\title{
ANALYTICITY OF SEMIGROUP ASSOCIATED WITH A LAMINATED COMPOSITE BEAM
}

\author{
Scott Hansen* \\ Department of Mathematics \\ lowa State University, Ames, IA 50011, USA \\ shansen@math.iastate.edu \\ Zhuangyi Liu \\ Department of Mathematics \& Statistics \\ University of Minnesota, Duluth, MN 55812, USA.
}

zliu@d.umn.edu

\begin{abstract}
We consider a system of coupled partial differential equations that describe the vibrations of laminated beam in which the layers are bonded together by a medium that dissipates energy at a rate proportional to the shear. We show that for the simplest model, in which only transverse inertial energy is accounted for, the associated semigroup is analytic.
\end{abstract}

\section{INTRODUCTION}

In this paper we study the damping characteristics of a laminated beam model introduced (for plates) in Hansen [3]. The model is derived under the assumption that the laminated beam consists of $2 n$ layers of Euler-Bernoulli beams bonded to one another by $2 n-1$ "adhesive layers" which resist shear, but otherwise have negligible physical characteristics. We suppose that damping is also included in the adhesive layers so that a force opposing shear, proportional to the rate of shear exists within the adhesive layers. No damping is included elsewhere in the model.

It was noticed in Hansen and Spies [5], that in the case of one adhesive layer, with constant coefficients and simply supported boundary conditions, the spectrum associated with the generator of the semigroup exhibits frequencyproportional damping characteristics (see Russell [16]). Many systems with this

\footnotetext{
*Research partially supported the National Science Foundation under grant DMS-9504822
}

The original version of this chapter was revised: The copyright line was incorrect. This has been corrected. The Erratum to this chapter is available at DOI: 10.1007/978-0-387-35359-3_40 
type of damping have been shown to be associated with analytic semigroups (e.g., Chen and Russell [1], Huang [8], Chen and Triggiani [2], Liu and Renardy [11], Liu and Yong [13], Liu and Liu [9]-[10], Hansen and Zhang [6], Lasiecka and Triggiani [12]).

We prove that analyticity indeed holds for the case of symmetrically layered laminated beams with an arbitrary number of layers in the case of simply supported boundary conditions. The proof is based on a contradiction argument similar to the one used in [13] to establish analyticity of another model. Our choice of simply supported boundary conditions allows us to apply the contradiction argument with the least amount of technical difficulties. However the basic approach applies to certain other boundary conditions as well.

This paper is organized as follows. Background on the modeling and wellposedness are given in Section 2. The analyticity of the multilayer case with simply-supported boundary conditions is proved in Section 3 .

\section{LAMINATED BEAMS AND PLATES}

The laminated beam model we consider are the beam-analogs of the laminated plate models described in Hansen [3] with viscous damping included in the adhesive layers. In the case of one adhesive layer this reduces to the beam model considered in Hansen and Spies [5].

We will consider the symmetric case in which the layers (both the beam layers and damping layers) of the laminated beam have material properties that are symmetric with respect to the centerline of the laminated beam. This allows for a decoupling of a set of "bending equations" that completely decouple from a set of "stretching equations". Without any symmetry assumptions, this decoupling does not exist and stronger damping would be needed to obtain energy decay due to longitudinal motions that are not damped.

\subsection{Modeling Assumptions}

In order to describe the meaning of the variables and physical constants it is necessary to describe briefly the modeling assumptions used in deriving the laminated beam model.

The laminated beam is assumed to consist of $2 n$ beam layers and $2 n-1$ adhesive layers that occupy the region $\Omega \times(-h / 2, h / 2)$ at equilibrium, where $\Omega=(0,1)$ for simplicity. Thus, since we are considering the symmetric case where all the material properties of the layers are symmetric with respect to the centerline of the laminated beam, this centerline must occur in an adhesive layer. (The case of an odd number of beam layers is similar.)

It is assumed in the modeling that (i) within each layer the longitudinal displacements vary linearly as a function of $x_{3}$, (ii) within each layer the transverse displacements are constant with respect to $x_{3}$, (iii) no slip is allowed on the interfaces, (iv) the Kirchhoff hypothesis (that normal sections remain normal after deformation) applies to the beam layers, (v) the adhesive layers resist 
shear but bending stresses and inertial forces are negligible, (vi) longitudinal kinetic evergy terms are assumed to be negligible.

To be more precise on assumption $(\mathrm{v})$, if $\phi_{i}$ denotes the shear variable for the $i$ th adhesive layer of thickness $d_{i}$, then $\phi_{i}=\delta_{i} / d_{i}+w_{x}$ where $w_{x}$ is the partial of the transverse displacement $w$ in the $x_{1}$ direction and $\delta_{i}$ is the change in longitudinal displacement of from the top to the bottom of the $i$ th adhesive layer. $\left(\delta_{i} / d_{i}\right.$ is also the rotation angle of the $i$ th adhesive layer.) The strain energy associated with this $i$ th layer is

$$
\mathcal{P}_{i}\left(K_{i}, d_{i}\right)=\frac{1}{2} \int_{\Omega} E_{i} d_{i}^{3} w_{x x}^{2}+12 E_{i} d_{i}\left(v_{i}\right)_{x}^{2}+K_{i} d_{i}\left(\phi_{i}\right)^{2} d x,
$$

where $K_{i}$ is the shear modulus of the $i$ th adhesive layer, $E_{i}$ is the Young's modulus of the $i$ th adhesive layer, and $v_{i}$ is the longitudinal displacement of this layer.

We formally take the limit as $K_{i} \rightarrow 0$ and $d_{i} \rightarrow 0$, but with

$$
\gamma_{i}:=\frac{K_{i}}{d_{i}} \text { fixed }
$$

What remains of the energy of the $i$ th adhesive layer is

$$
\mathcal{P}_{i}=\frac{1}{2} \int_{\Omega} \gamma_{i} \delta_{i}^{2} d x .
$$

Due to the symmetry of motion, the energy in the $i$ th layer (either adhesive or beam) above the centerline equal to that of the $i$ th one below the centerline. This also applies to the center adhesive layer, provided we regard this layer as two consecutive adhesive layers with half the original thickness. This convention does not introduce additional degrees of freedom since we are restricting our interest to bending solutions, which are antisymmetric with respect to the centerline of the beam.

Define the following $n$ by $n$ matrices

$$
\begin{array}{ll}
\mathbf{g}=\operatorname{diag}\left(\gamma_{1}, \gamma_{2}, \ldots, \gamma_{n}\right) & \mathbf{p}=\operatorname{diag}\left(\rho_{1}, \rho_{2}, \ldots, \rho_{n}\right) \\
\mathbf{h}=\operatorname{diag}\left(h_{1}, h_{2}, \ldots, h_{n}\right) & \mathbf{D}=\operatorname{diag}\left(D_{1}, D_{2}, \ldots, D_{n}\right)
\end{array}
$$

where $\left\{\gamma_{i}\right\}$ are the previously described elastic parameters, $h_{i}$ is the thickness of the $i$-th beam layer, $D_{i} h_{i}^{3}$ is the modulus of flexural rigidity for the $i$ th beam layer, $\rho_{i}$ is the density of the $i$ th beam layer.

If $M$ and $N$ are matrices in $\mathbf{R}^{m n}$, by $M \cdot N$ we mean the natural scalar product in $\mathbf{R}^{m n}$. We denote $(\theta, \xi)_{\Omega}=\int_{\Omega} \theta \cdot \xi d x_{1}$.

Also define $M$ to be the $n \times n$ matrix with 1 's on and below the main diagonal and 0 's elsewhere and let $\overrightarrow{1}$ denote the column vector consisting of $n 1$ 's. We can express $v$ is terms of $\psi$ and $\delta$ as

$$
v=M \delta-(M-I / 2) \mathbf{h} \overrightarrow{1} w_{x}=: M \delta-\mathbf{c} w_{x} .
$$


The energy we use is $\mathcal{E}(t)=c(\dot{w})+a(w, \delta)$ where

$$
\begin{array}{ll}
c(w ; w) & =(m w, w)_{\Omega} \\
a(w, \delta ; w, \delta) & =\left(D_{t o t} w_{x x}, w_{x x}\right)_{\Omega}+12\left(\mathbf{D h} v_{x}, v_{x}\right)_{\Omega}+(\mathbf{g} \delta, \delta)_{\Omega}
\end{array}
$$

where

$$
m=\sum_{i=1}^{n} \rho_{i} h_{i}, \quad D_{t o t}=\sum_{i=1}^{n} D_{i} h_{i}^{3}
$$

and $v$ is defined as in (2.1). Hamilton's principle can now be used to derive a conservative system of equations. (The system one obtains is the same as equations (23)-(24) of [3], but without the rotational inertia terms in (23).) Once this is done, damping can be included by the correspondence principle. Since we wish to include shear damping in the adhesive layers, to each $\gamma_{i}$ is a corresponding damping parameter $\beta_{i}$, and hence corresponding to the diagonal stiffness matrix $\mathrm{g}$ is a corresponding viscosity matrix $\mathbf{b}=\operatorname{diag}\left(\beta_{1}, \beta_{2}, \ldots, \beta_{n}\right)$ that we include in the system of equations via the correspondence $\mathbf{g} \delta \rightarrow \mathbf{g} \delta+$ $\mathbf{b} \delta_{t}$.

The final system of equations obtained is the following.

$$
\begin{gathered}
m \ddot{w}+\left(D_{t o t} w_{x x}\right)_{x x}-12 \mathbf{c}^{T}\left(\mathbf{D h} v_{x x}\right)_{x}=0 \quad x \in \Omega, t>0 \\
-12 M^{T}\left(\mathbf{D h} v_{x}\right)_{x}+\mathbf{g} \delta+\mathbf{b} \dot{\delta}=0 \quad x \in \Omega, t>0
\end{gathered}
$$

where $v$ is defined in (2.1).

The simply supported boundary conditions are

$$
w=0, \quad w_{x x}=0, \quad \delta_{x}=0, \quad x=0,1 .
$$

\subsection{Well-posedness}

We let

$$
\mathcal{V}=\left(H_{0}^{1} \cap H^{2}\right)(\Omega) \times\left(H^{1}(\Omega)\right)^{n}, \quad \mathcal{H}=L^{2}(\Omega)
$$

with norms defined by

$$
\|\{w, \delta\}\|_{\mathcal{V}}^{2}=a(w, \delta ; w, \delta), \quad\|y\|_{\mathcal{H}}^{2}=c(y ; y)
$$

The forms $a(\cdot ; \cdot)$ and $c(\cdot ; \cdot)$ can be shown to be continuous, symmetric, bilinear and positive definite on $\mathcal{V} \times \mathcal{V}$ and $\mathcal{H} \times \mathcal{H}$ respectively. (See [4] for proof of a similar situation.) Therefore these forms extend to complex inner products on $\mathcal{V}$ and $\mathcal{H}$ respectively. We define the energy space $\mathcal{E}$ by

$$
\mathcal{E}=\{(w, y, \delta):(w, \delta) \in \mathcal{V}, y \in \mathcal{H}\}
$$

with the inner product $\langle\cdot, \cdot\rangle_{\mathcal{E}}$ that corresponds to the forms $a(\cdot ; \cdot)$ and $c(\cdot ; \cdot)$.

We let $y=\dot{w}$ and rewrite the equations of motion (2.3)-(2.4) as

$$
\frac{d}{d t}\left(\begin{array}{l}
w \\
y \\
\delta
\end{array}\right)=\mathcal{A}\left(\begin{array}{l}
w \\
y \\
\delta
\end{array}\right):=\left(\begin{array}{l}
y \\
\frac{1}{m}\left(-\left(D_{t o t} w_{x x}\right)_{x x}+12\left(\mathbf{c}^{T} \mathbf{h D} v_{x}\right)_{x x}\right) \\
\mathbf{b}^{-1}\left(12 M^{T} \mathbf{h}\left(\mathbf{D} v_{x}\right)_{x}-\mathbf{g} \delta\right)
\end{array}\right)
$$


For the moment, let use $\mathcal{A}(\mathbf{b})$ to denote the dependence of $\mathcal{A}$ on the damping vector $\mathbf{b}$. A calculation reveals that the adjoint $\mathcal{A}^{*}$ of $\mathcal{A}$ is given by

$$
\mathcal{A}^{*}=-\mathcal{A}(-\mathbf{b})
$$

and that for $(w, y, \delta) \in \mathcal{D}(\mathcal{A})$, (for brevity, we omit a precise description of $\mathcal{D}(\mathcal{A})$ here $)$

$\operatorname{Re}<\mathcal{A}\left(\begin{array}{l}w \\ y \\ \delta\end{array}\right),\left(\begin{array}{l}w \\ y \\ \delta\end{array}\right)>=\operatorname{Re}<\mathcal{A}^{*}\left(\begin{array}{l}w \\ y \\ \delta\end{array}\right),\left(\begin{array}{c}w \\ y \\ \delta\end{array}\right)>=-<\mathbf{b}^{-1} \xi, \xi>_{L^{2}}$,

where $\xi=M^{T} \mathbf{h}\left(\mathbf{D} v_{x}\right)_{x}-g \delta$.

It follows that both $\mathcal{A}$ and $\mathcal{A}^{*}$ are dissipative. Since $A$ is easily seen to be densely defined and closed, by a corollary to the Lumer-Phillips theorem ([14], p.15), we have the following.

Proposition 2.1 The operator $\mathcal{A}$ is the generator of a $C_{0}$ semigroup of contractions on $\mathcal{V} \times \mathcal{H}$. Consequently, given any initial data $\left\{w_{0}, \delta_{0}\right\} \in \mathcal{V}$ and $w_{1} \in \mathcal{H}$, there is a unique solution $\{w, \delta\}$ for the equations of motion with

$$
\{w, \delta\} \in C([0, \infty) ; \mathcal{V}), \quad w_{t} \in C([0, \infty) ; \mathcal{H})
$$

\section{DECAY RESULTS}

We show that $\mathcal{A}$ is the generator of an analytic semigroup.

Proposition 3.1 Assume that each $b_{i}, i=1,2, \ldots n$ is positive. Then the operator $\mathcal{A}$ is the generator of an analytic semigroup.

Proof: We employ a contradiction argument for the analyticity of a $C_{0}$ semigroup of contractions proposed in [13].

From Pazy [14], we have that $\left\{e^{t \mathcal{A}}\right\}$ is analytic if and only if $\left\|\lambda(A-\lambda I)^{-1}\right\|$ is uniformly bounded for all $\lambda$ on some vertical line. We will show that this in fact holds on the imaginary axis. Equivalently, we will show that there exists $\epsilon>0$ such that

$$
\inf _{\|z\|=1}\|(\mathcal{A}-i s I) z\| \geq \epsilon|i s| \quad \forall s \in \mathbf{R} .
$$

If, on the contrary, this condition does not hold then there exists $\left(s_{n}\right) \in \mathbf{R}$ and $z_{n}=\left\{w_{n}, \dot{w}_{n}, \delta_{n}\right\}$ with $\left\|z_{n}\right\|_{\mathcal{E}}=1$ such that

$$
\lim _{n \rightarrow \infty}\left\|i z_{n}-\frac{1}{s_{n}} \mathcal{A} z_{n}\right\|_{\mathcal{E}}=0 .
$$

Due to the fact that the norm of the resolvent is symmetric with respect to the real axis we can assume that each $s_{n}$ is positive. Furthermore, one can directly show (using the dissipativity of $\mathcal{A}$ and compactness of the resolvent) that $\mathcal{A}$ has no spectrum on the imaginary axis. Thus we may assume that $s_{n} \rightarrow+\infty$. 
Let us use the notation $\phi^{\prime}=\frac{d}{d x} \phi, \phi^{\prime \prime}=\frac{d^{2}}{d x^{2}} \phi$ and so forth. Explicitly writing out (3.8) gives

$i\left(\begin{array}{l}w_{n} \\ y_{n} \\ \delta_{n}\end{array}\right)-s_{n}^{-1}\left(\begin{array}{l}y_{n} \\ m^{-1}\left[-\left(D_{t o t} w_{n}^{\prime \prime}\right)^{\prime \prime}+12\left(\mathbf{c}^{T} \mathbf{h D} v_{n}^{\prime}\right)^{\prime \prime}\right] \\ \mathbf{b}^{-1}\left[-12 M^{T} \mathbf{h}\left(\mathbf{D} v_{n}^{\prime}\right)^{\prime}+\mathbf{g} \delta\right]\end{array}\right)=:\left(\begin{array}{l}\xi_{1}(n) \\ \xi_{2}(n) \\ \xi_{3}(n)\end{array}\right) \longrightarrow 0$

as $n \rightarrow \infty$. The convergence is in the energy space, which implies

$$
\begin{aligned}
\xi_{2} \rightarrow 0 & \text { in } L^{2}(\Omega) \\
\xi_{1}^{\prime \prime} \rightarrow 0 & \text { in } L^{2}(\Omega) \\
\xi_{3} \rightarrow 0 & \text { in } L^{2}(\Omega) \\
\left(M \xi_{3}-\mathrm{c} \xi_{1}^{\prime}\right)^{\prime} \rightarrow 0 & \text { in } L^{2}(\Omega)
\end{aligned}
$$

On the other hand, from (3.8) we have

$$
\operatorname{Re}\left\langle\left(i-s_{n}^{-1} \mathcal{A}\right) z_{n}, z_{n}\right\rangle_{\mathcal{E}} \rightarrow 0 \quad \text { as } n \rightarrow \infty
$$

By a direct calculation one finds that

$$
\begin{aligned}
\operatorname{Re}\left\langle\left(i-s_{n}^{-1} \mathcal{A}\right) z_{n}, z_{n}\right\rangle_{\mathcal{E}} & =-s_{n}^{-1} \operatorname{Re}\left\langle\mathcal{A} z_{n}, z_{n}\right\rangle_{\mathcal{E}} \\
& =-s_{n}^{-1}\left\|\mathbf{b}^{-1 / 2}\left(-12 M^{T} \mathbf{h}\left(\mathbf{D} v_{n}^{\prime}\right)^{\prime}+\mathbf{g} \delta_{n}\right)\right\|_{L^{2}}^{2},
\end{aligned}
$$

and hence since $\mathbf{b}$ is invertible we obtain

$$
s_{n}^{-1}\left\|-12 M^{T} \mathbf{h}\left(\mathbf{D} v_{n}^{\prime}\right)^{\prime}+\mathbf{g} \delta_{n}\right\|_{L^{2}}^{2} \rightarrow 0 .
$$

Substituting this into (3.12) gives

$$
\left\|\delta_{n}\right\|_{L^{2}} \rightarrow 0
$$

and since also $\|\mathbf{g} \delta\|_{L^{2}} \rightarrow 0,(3.14)$ also implies (using the invertibility of $M, \mathbf{h}$ ) that

$$
s_{n}^{-1}\left\|\left(\mathbf{D} v_{n}^{\prime}\right)^{\prime}\right\|_{L^{2}}^{2} \rightarrow 0 \text {. }
$$

Since $\left\|\xi_{1}^{\prime \prime}\right\|_{L^{2}} \rightarrow 0,(3.13)$ is equivalent to $\xi_{3}^{\prime} \rightarrow 0$ in $L^{2}$, or, since (3.12) already holds,

$$
i \mathbf{b} \delta_{n}-s_{n}^{-1}\left(-12 M^{T} \mathbf{h}\left(\mathbf{D} v_{n}^{\prime}\right)^{\prime}+\mathbf{g} \delta_{n}\right) \rightarrow 0 \text { in } H^{1}(\Omega) .
$$

Note that $\left(w_{n}^{\prime \prime}\right)$ is bounded in $L^{2}$ and therefore by (3.11)

$$
\left(s_{n}^{-1} y_{n}^{\prime \prime}\right) \text { bounded in } L^{2} \text {. }
$$

Since $\left(y_{n}\right)$ is also bounded, by interpolation (or integration by parts and Schwartz inequality) we have

$$
\left(s_{n}^{-1 / 2} y_{n}^{\prime}\right) \quad \text { bounded in } L^{2}(\Omega)
$$


We take the inner product of (3.13) with $\mathbf{D} v_{n}^{\prime}$ in $L^{2}(\Omega)$ to obtain

$$
\begin{array}{r}
i<\mathbf{D} v_{n}^{\prime}, \mathbf{D} v_{n}^{\prime}>+<M \mathbf{b}^{-1}\left[-12 M^{T} \mathbf{h}\left(\mathbf{D} v_{n}^{\prime}\right)^{\prime}+\mathbf{g} \delta\right]-\mathbf{c} y_{n}^{\prime}, s_{n}^{-1}\left(\mathbf{D} v_{n}^{\prime}\right)^{\prime}> \\
=i<\mathbf{D} v_{n}^{\prime}, \mathbf{D} v_{n}^{\prime}>+<M \mathbf{b}^{-1}\left[-12 M^{T} \mathbf{h}\left(\mathbf{D} v_{n}^{\prime}\right)^{\prime}\right], s_{n}^{-1}\left(\mathbf{D} v_{n}^{\prime}\right)^{\prime}> \\
\quad+<M \mathbf{b}^{-1} \mathbf{g} \delta_{n}, s_{n}^{-1}\left(\mathbf{D} v_{n}^{\prime}\right)^{\prime}>-<s_{n}^{-1 / 2} \mathbf{c} y_{n}^{\prime}, s_{n}^{-1 / 2}\left(\mathbf{D} v_{n}^{\prime}\right)^{\prime}>
\end{array}
$$

where the last three terms go to zero by $(3.15),(3.16)$ and (3.19). Therefore

$$
\left\|v_{n}^{\prime}\right\|_{L^{2}} \rightarrow 0
$$

Now substitute (3.23) in (3.13) to obtain

$$
s_{n}^{-1}\left(M \mathbf{b}^{-1}\left[-12 M^{T} \mathbf{h}\left(\mathbf{D} v_{n}^{\prime}\right)^{\prime}+\mathbf{g} \delta\right]-\mathbf{c} y_{n}^{\prime}\right)^{\prime} \rightarrow 0 .
$$

Next we take the inner product of $(3.24)$ with $\overrightarrow{1} y_{n}$, using the facts that by (3.15), (3.16), (3.19)

$$
<s_{n}^{-1 / 2}\left(D v_{n}^{\prime}\right)^{\prime}, s_{n}^{-1 / 2} \overrightarrow{1} y_{n}^{\prime}>\rightarrow 0, \quad<\delta_{n}, s_{n}^{-1} \overrightarrow{1} y_{n}^{\prime}>\rightarrow 0
$$

to obtain that $s_{n}^{-1}\left\|\mathbf{c} y_{n}^{\prime}\right\|_{L^{2}}^{2} \rightarrow 0$ and hence

$$
s_{n}^{-1 / 2}\left\|y_{n}^{\prime}\right\|_{L^{2}} \rightarrow 0 \text {. }
$$

Take the inner product of (3.10) with $m y_{n}$ to obtain

$$
i m\left\|y_{n}\right\|^{2}+s_{n}^{-1} 12<\mathbf{c}^{T} \mathbf{h}\left(\mathbf{D} v_{n}^{\prime}\right)^{\prime}, y_{n}^{\prime}>+s_{n}^{-1}<D_{t o t} w_{n}^{\prime \prime}, y_{n}^{\prime \prime}>\rightarrow 0 .
$$

The second term goes to zero due to $(3.16),(3.26)$ and the third term goes to zero due to $(3.18)$ and the boundedness of $\left(D_{t o t} w^{\prime \prime}\right)$ in $L^{2}$. Hence

$$
\left\|y_{n}\right\|_{L^{2}}^{2} \rightarrow 0 \text {. }
$$

Now calculate the inner product of (3.11) with $D_{t o t} w_{n}^{\prime \prime}$ and subtract from this the inner product of (3.10) with $y_{n}$. After some integrations by parts we obtain

$$
i<D_{t o t} w_{n}^{\prime \prime}, w_{n}^{\prime \prime}>-i<m y_{n}, y_{n}>-s_{n}^{-1}<12\left(\mathbf{c}^{T} \mathbf{h D} v_{n}^{\prime}\right)^{\prime}, y_{n}^{\prime}>\rightarrow 0
$$

where the last term goes to zero by (3.16) and (3.26). It follows from (3.28) that

$$
\left\|w_{n}^{\prime \prime}\right\|_{L^{2}} \rightarrow 0
$$

Combining (3.29) with (3.15),(3.23), and (3.28) we have that $\left\|z_{n}\right\|_{\mathcal{E}} \rightarrow 0$ as $n \rightarrow \infty$, which is a contradiction.

In the preceeding proof we have shown that $\left\|\lambda(\mathcal{A}-\lambda I)^{-1}\right\|$ is uniformly bounded on the imaginary axis. Hence, since zero is in the resolvent set, we also have that $\left\|(\mathcal{A}-\lambda I)^{-1}\right\|$ is bounded on the imaginary axis. Therefore, using [7], as a corollary of Proposition 3.1 we have the following result.

Corollary 3.1 The semigroup $e^{t \mathcal{A}}$ of Proposition 3.1 is exponential stable, i.e., there exists $\epsilon>0$ and $M>1$ such that

$$
\left\|e^{t \mathcal{A}} z\right\|_{\mathcal{E}} \leq M e^{-\epsilon t}\|z\|_{\mathcal{E}} .
$$




\section{References}

[1] Chen, G. and Russell, D. L. (1981/1982). A mathematical model for linear elastic systems with structural damping. Quart. Appl. Math., 39:433-454.

[2] Chen, S. and Triggiani, R. (1989). Proof of extensions of two conjectures on structural damping for elastic systems, the case $\frac{1}{2} \leq \alpha \leq 1$. Pacific J. Math., 39:15-55.

[3] Hansen, S. (1998). Modeling and analysis of multilayer laminated plates. to appear: ESAIM Proc. "Control and Partial Differential" Conference at CIRM, Marseille-Luminy, 16-20 jun 1997.

[4] Hansen, S. (1997). A dynamical model for multilayered plates with independent shear deformations Quart. Appl. Math. LV:601-621

[5] Hansen, S. and Spies, R. (1997) Structural damping in laminated beams due to interfacial slip. J. Sound Vib., 204:183-202

[6] Hansen, S. and Zhang, B-Y. (1997) Boundary control of a linear thermoelastic beam. JMAA, 210:182-205

[7] Huang, F. (1985) Characteristic conditions for the exponential stability of linear dynamical systems in Hilbert spaces. Ann. of Diff. Eqs., 1:43-56

[8] Huang, F. (1988). On the mathematical medel for linear elastic systems with analytic damping. SIAM J. Control and Optimization, 26:714-724.

[9] Liu, K. and Liu, Z. (1997). Exponential Stability and Analyticity of the abstract thermoelastic evolution equation. Z. agnew Math. Phys, 48:885904.

[10] Liu, K. and Liu, Z. (1997). Analyticity and differentiability of semigroups associated with elastic systems with damping and gyroscopic forces. $J$. Diff. Eqns., Vol. 141, 2:340-355.

[11] Liu, Z. and Renardy, M. (1995). A note on the equations of a thermoelastic plate. Appl. Math. Lett., Vol. 8, 3:1-6.

[12] Lasiecka, I. and Triggiani, R. (1998). Two direct proofs on the analyticity of the S.C. semigroup arising in abstract thermo-elastic equation. Adv. Diff. Eqns., to appear.

[13] Liu, Z. and Yong, J. (1998) Qualitative properties of certain $C_{0}$ semigroups arising in elastic systems with various dampings. Adv. Diff. Eqns., to appear.

[14] Pazy, A. (1983). Semigroups of Linear Operators and Applications to Partial Differential Equations. Springer, New York.

[15] Russell, D. L. (1991). A comparison of certain elastic dissipation mechanisms via decoupling and projection techniques. Quarterly of Appl. Math., Vol. XLIX, 2:373-396.

[16] Russell, D. L. (1992) On mathematical models for the elastic beam with frequency- proportional damping. Control and Estimation of Distributed Parameter Systems, H. T. Banks ed., SIAM, Philadelphia. 